\title{
A CASE OF LYMPHOSARCOMA OF THE INTESTINE IN A CHILD
}

\author{
BY \\ KATHARINE J. GUTHRIE, M.D. \\ (From the Department of Pathology, Royal Hospital for Sick Children, Glasgow)
}

Lymphosarcoma of the intestine is a rare disease in children and especially in those of British race, to judge from the dearth of case reports in this country, but the incidence appears to be higher in America since it is the American literature that furnishes most of the examples published in the past fifty years.

The present case is reported since it combines unusual intestinal and renal lesions.

\section{Case Report}

The patient was a boy aged $10 \frac{3}{4}$ years admitted to the Royal Hospital for Sick Children, Glasgow, on Sept. 29, 1944. Tonsillectomy at the age of five years and measles and chickenpox in early childhood were the only items of note in the history, and he was an active, intelligent boy before the present illness which began some six weeks before death with pallor and listlessness, accompanied by pain in the knees and face. A fortnight later he had a single transient attack of abdominal pain without vomiting, of which there are no available particulars. His face shortly afterwards began to swell, and $x$-ray examination revealed tumour-like nodules in the skull. From about mid-September onwards he was confined to bed suffering from headache, sleeplessness, anorexia, and weakness. The bowels were regular throughout, and there was no dysuria.

On admission to hospital he was a pale, emaciated, acutely ill child with marked muscle wasting, loss of tendon jerks, and pitting oedema of the ankles. The abdomen was swollen and tense, the superficial veins were dilated, and a large, firm, nodular mass, tender to palpation, was present in the hypogastrium and right iliac fossa. The kidneys and spleen could not be defined but the smooth edge of the slightly enlarged liver was palpable below the costal margin. No shifting dullness. was elicited, and there was no visible peristalsis. The lungs and heart were normal. Soft swellings were found over the vertex of the skull and in the lower jaw. There was a right-sided facial paralysis and the left eye was slightly proptosed. Both pupils reacted to light and on accommodation, and the fundi were normal. His blood on the day after admission had 58 per cent. haemoglobin, a red cell count of approximately 5,000,000 per c.mm. of blood, and a leucocytosis of some 16,000 per c.mm. Ring staining was the only abnormality in the red cells. On differential count neutrophile polymorphs numbered 57.5 per cent., lymphocytes 33 per cent., monocytes 6 per cent., and immature cells of myeloblastic type 3.5 per cent. The urine was examined several times and showed merely a trace of albumin, with a few granular casts and leucocytes. The bowels were regular. There was rapid deterioration in the child's condition with increasing asthenia, and death occurred five days after admission.

Autopsy. An autopsy was performed next day. The subject was well grown for his age but pale and extremely emaciated. There was no noteworthy enlargement of the glands in the neck, axillae, and groins. The long bones were all normal in contour, but swellings were palpable in the vault of the skull, and a nodule composed of pale, soft, tumour tissue was present in the left lower jaw in the gap left by two missing premolar teeth. The soft tissues but not the bone were invaded. No appreciable proptosis of the left eye was observed, and periorbital haemorrhage was absent. No tumour deposits were seen in the atrophic thymus, in the mediastinal tymph glands, or in the lungs. Both pleural sacs were normal. Many small, pale, sub-epicardial tumour nodules were present anteriorly in the normal-sized heart, projecting slightly above the surface. The scanty pericardial fluid was pale yellow and clear.

Below the diaphragm the picture was striking owing to the presence of large tumours which involved the abdominal glands, the mesentery, omentum, kidneys, and intestines. The root of the mesentery was occupied by a bulky, nodular neoplasm composed of fused mesenteric glands; a few nodes at the periphery remained discrete and these were greatly enlarged. On section of this tumour the cut surface was pale and homogeneous and the texture soft and brain-like. A smaller glandular mass of similar type and showing central haemorrhage was present in the ileo-caecal angle. In addition, the entire mesentery was loaded along the course of the lymphatics with innumerable small, discrete tumour-bearing glands. There was widespread infiltration of the omentum with great increase in bulk. The stomach itself was of normal size and contained only three small, flat, sessile 
tumour nodules on the mucosa, two near the cardia and one on the lesser curvature close to the pylorus. It was, however, in the lower gastro-intestinal tract that tumour growth was most exuberant. The duodenum was tumour-free, but from the duodenojejunal junction to within a few feet of the terminal ileum the wall of the small bowel was heavily loaded with tumour tissue which had infiltrated especially the circular folds of the mucosa and had expanded them into thick, prominent ridges. The Peyer's patches were converted into large flat plaques, some showing superficial ulceration. All the tumour nodules were sessile and by their annular mode of spread had stretched the bowel so that the lumen was widely patent and was not obstructed (fig. 1). Some six feet above the ileocaecal valve, tumour growth ceased rather abruptly, and the terminal ileum was normal in appearance and calibre.

A massive tumour, which was regarded as the primary growth, occupied the caecum with great thickening of the wall, but central ulceration had taken place and here again there was no obstruction. Intramural haemorrhage with necrosis had occurred, and rupture was imminent, but there was no trace of peritonitis to indicate antemortem leakage of intestinal contents. The rest of the large bowel was normal in all respects.

The kidneys (fig. 2) presented a remarkable appearance from their resemblance to large bilateral renal tumours, though retaining their reniform shape and smooth surface. In fact, they consisted chiefly of neoplastic tissue which permeated the organs in all parts and replaced their normal structure by pale, homogeneous tissue flecked by small haemorrhages. The cortex on each side was greatly widened, and overgrowth of the interpyramidal zone had forced apart the small, pale-red calyces. The capsule on each side was infiltrated and thickened by tumour growth. Both renal pelves and ureters were normal. The spleen, liver, adrenal glands, pancreas, and gallbladder were all free from metastases.

Widespread skeletal deposits were present, notably in the bones forming the vault of the skull, where nodules of varying size arose both in the inner and outer tables. A mass growing from the left parietal bone projected inwards and indented the underlying cerebral cortex in the motor area, thus accounting for the right-sided facial paralysis observed during life, but there was no invasion of the brain or meninges. No tumour nodules were found in the base of the skull or in the orbital cavities. Metastases were present in several of the lower dorsal vertebrae, and one or two ribs had minute, flat, button-like nodules on the periosteum, but the pelvic bones were tumour-free. The marrow cavity of the right femur was filled by pale, tumour-like tissue, but there was no sub-periosteal infiltration.

Histology. Paraffin sections were prepared and stained by a variety of methods, including haematoxylin and eosin, iron haematoxylin and van
Gieson, the Romanowsky method published by Barrett (1944) and the reticulin stain described by Lendrum (1947).

The primary growth and the secondary deposits in various tissues showed an identical homogeneous structure consisting almost entirely of lymphoblastic cells. The individual tumour cells were round or oval with a relatively large, pale, vesicular nucleus surrounded by a narrow basophile-staining protoplasmic rim. The nuclear chromatin formed a delicate lightly-stippled network which stained much less densely than in the mature lymphocyte. A single nucleolus was frequently present but in many cells none could be demonstrated by any staining method. Mitoses were fairly numerous but multinucleated giant cells were scanty and there was no noteworthy inflammatory cell component. There was no attempt at follicle formation and the cells were arranged in the form of a loose mosaic. Collagenous stroma was scanty and there was little attempt at reticulin formation on the part of the tumour cells which appeared rather to have invaded and destroyed the normal pre-formed reticulin than to be laying down new fibrils. This marked capacity to invade and destroy all normal tissues in its advancing path was a feature of the growth. It was well supplied with thin-walled blood vessels and there were few areas of necrosis.

Sections of the caecal tumour (fig. 3) showed massive invasion of the mucosa and submucosa. These coats were densely packed with lymphoblasts and greatly thickened, but it was still possible to distinguish the muscularis mucosae. The delicate processes of the mucous membrane were converted into massive, club-like structures, many necrotic at the tip, and the glands of Lieberkühn were widely separated, small, and atrophic, while some had undergone cystic degeneration. Both the circular and the longitudinal muscle coats, which were of about the normal width, were extensively infiltrated and fragmented by the invading tumour cells, and these had reached the serous surface. In some of the smaller lymphoid nodules in the small intestine, tumour infiltration was confined to the mucous and submucous coats. In the kidneys the capsule was thickened by tumour growth and the degree of interstitial invasion was extreme with almost complete destruction of the glomeruli and tubules in some areas. Surviving renal structures were small, atrophic, and isolated in a mass of lymphoblasts (fig. 4). Other portions of the cortex were fairly well preserved, but even in such relatively intact areas tumour cells were present in smaller numbers and had penetrated between the tubules. By contrast, the medullary zone was relatively tumour-free. In the lymph glands the normal structure was replaced by diffuse cellular infiltration without follicular arrangement. Skeletal metastases were of similar uniform type, with replacement of the heterogeneous components of the normal marrow by the homogeneous tumour cells. Nodules in the heart wall were composed of dense, lympho- 


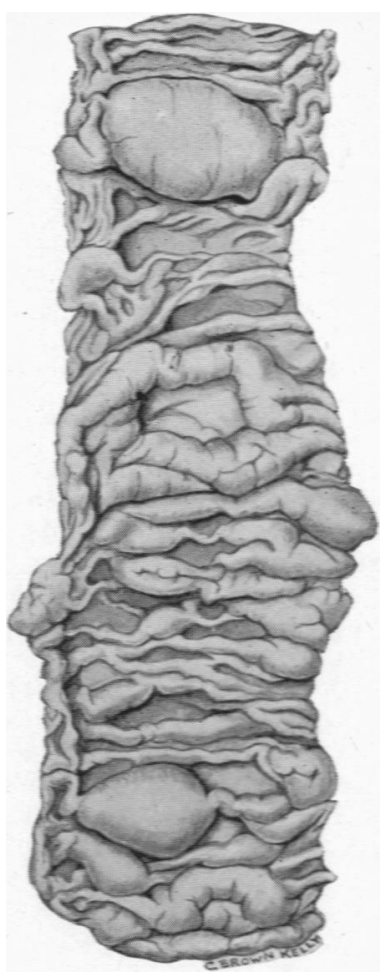

Fig. 1.-Dilated small bowel loaded with nodules of lymphosarcoma. (Half natural size.)

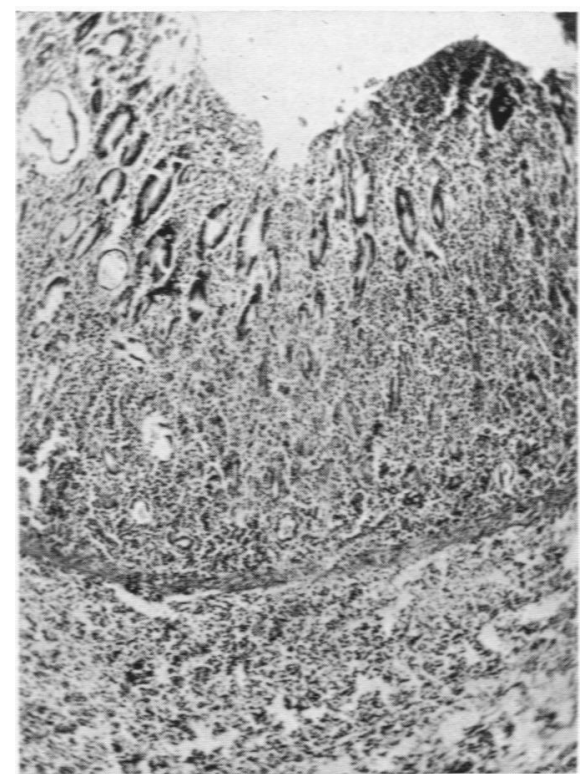

Fig. 3.-Caecum permeated by lymphoblastic cells with great thickening of mucous and submucous coats. $(\times 15$.)

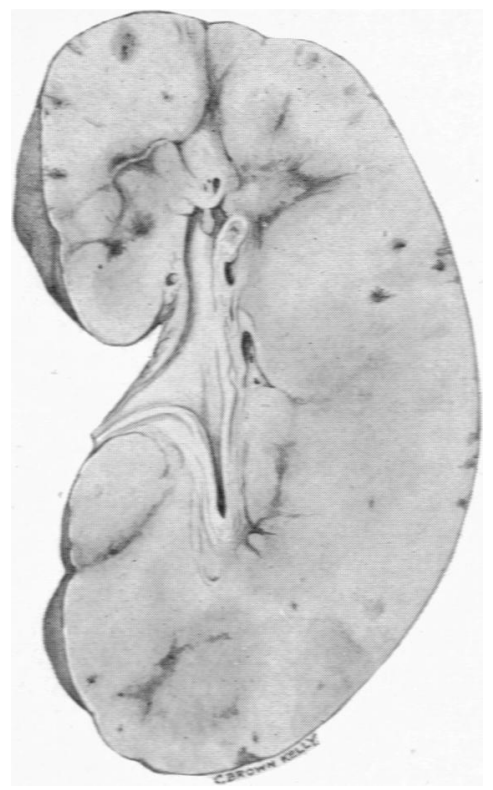

Fig. 2.-Enlarged kidney showing diffuse lymphosarcomatous infiltration. (Half natural size.)

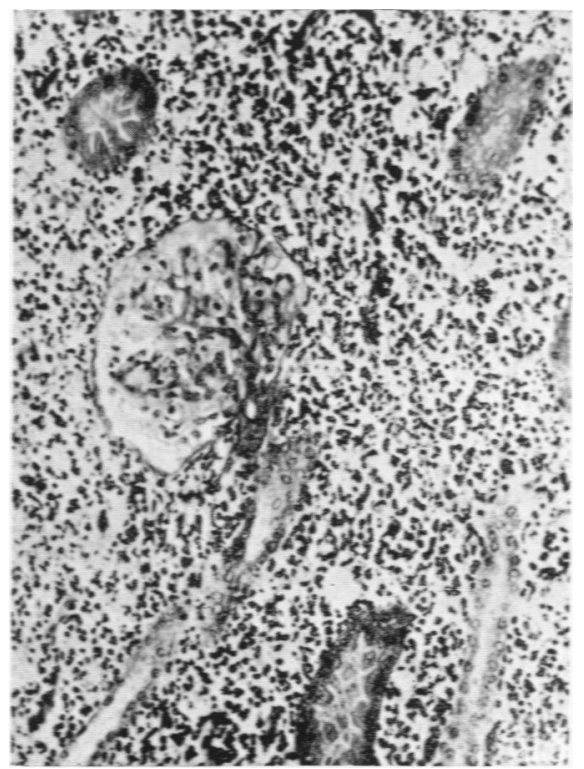

Frg. 4.-Kidney showing diffuse interstitial infiltration by tumour cells with few surviving glomeruli and tubules. $(\times 180$. $)$ 
blastic collections which had invaded the epicardium and spread along the interstitial planes of the myocardium with loss of the muscle fibres.

\section{Discussion}

Since Ewing (1913) first suggested the derivation of certain lymphoid tumours from reticulum cells, the term reticulosarcoma has been employed with increasing frequency in the literature. A detailed classification is given by Robb-Smith (1938) of the various types of tumour arising in reticulum cells at all transitional stages from the primitive syncytial reticulum to the mature lymphocyte. Willis (1948), however, regards these several types of tumour not as rigidly distinct species, but as related variants of a single species, and would restrict the term reticulosarcoma to tumours where differentiation of reticular tissue is distinct and predominant. In the present case the lymphoblastic cells composing the tumour were deficient in reticulin formation, and it has been classified accordingly as a lymphosarcoma of lymphoblastic type rather than as a reticulosarcoma. Uniformity of cytological structure is characteristic of lymphosarcoma. Boyd (1947) regards this feature as outstanding, and Willis describes the structure as composed of " diffuse masses of rounded cells of uniform size. . . . The cells penetrate into all the available interstices of the invaded tissue, whether kidney, liver, intestine, heart wall, lung or lymphoid tissue itself, mingling inextricably with the surviving components of these.'

Intestinal lymphosarcoma is rare at all ages. Warthin (1915) found only two examples in an analysis of 2,000 malignant neoplasms, and it is even more uncommon in early than in later life, although youth and even infancy are not entirely exempt. A congenital case is recorded by Stern (1901), and Ewing (1922) states, ' Not a few lymphosarcomas arise in infants and appear to have a congenital basis.'

The disease is twice or thrice as common in males as in females. Much of the literature on intestinal sarcoma, both old and recent, is of American origin. Thus, Libman (1900) reports five cases of his own, two in children, aged respectively $3 \frac{1}{2}$ and 12 years. This author also collected from the literature fifty-one cases, of which six occurred in the first and nine in the second decade. Jopson and White (1901), also in America, describe a case in a child aged 4 years. Cutler et al. (1945) of the Harvard Medical School, record five cases arising primarily in the bowel in children aged respectively $4,21,64$ and 66 months, and 7 years. They state that neoplasms of the small and large intestine are extremely rare in children as compared with adults. Simpson-Smith (1938), who publishes one of the few British series, found only six examples of abdominal sarcomata in the records of the London Hospital for Sick Children dating back to 1900 , and on making an exhaustive search of the world literature as far back as 1852 he discovered only a hundred similar cases in children, the maximum incidence falling between the ages of 1 and 5 years, with five cases in infancy and two of these in the neonatal period. Of these 106 tumours, seventy involved the small intestine and most commonly the ileum; thirteen arose in the caecum, six in the appendix, and the rest in various parts of the colon. Ewing (1922) and Ullman and Abeshouse (1932) also found the ileum the site of predilection. Willis remarks that in cases presenting as generalized lymphosarcomatosis the site of origin is not always clear, but where a large tumour of a particular region is present this suggests, but does not always prove, that the tumour began in this region. In our case the large, nodular mass in the caecum, palpable during life, is believed to represent the primary growth, since by analogy with the sequence in abdominal tuberculosis, where infection spreads from the bowel along the mesenteric lymphatics, it appears more likely that the tumour had originated in the intestine and extended along the lymph paths draining into the root glands, than that it was primarily glandular with retrograde spread to the bowel.

Rapid and extensive metastasis is the rule in lymphosarcoma since this type of tumour is characteristically of multicentric origin, arising not in a single lymph node, but simultaneously in groups of nodes or in multiple lymphoid foci in a mucous membrane like that of the small bowel, and is capable of spreading with great rapidity along submucous surfaces. In the later stages dissemination occurs also by way of the lymphatics and the blood stream. In the case under discussion lymphatic spread in the mesentery was clearly demonstrated, while skeletal metastases were undoubtedly blood-borne.

The disease is seldom arrested, and death generally occurs within a few months of onset. Occasionally its clinical course is hyper-acute, not exceeding twenty-four hours. Thus in three of Libman's five cases the clinical picture suggested appendicitis. In one of these there was only a single day's history of right-sided abdominal pain, and laparotomy revealed a perforated jejunal sarcoma with escape of intestinal contents. Ewing (1922) quotes BirchHirschfeld's case, rapidly fatal, which followed typhoid fever and was practically a continuation of typhoid hyperplasia. In Simpson-Smith's series of 106 juvenile cases, over 60 per cent. died within six months, and 92 per cent. within one year of diagnosis. Munson and Marx (1946), however, believe that the outlook is apparently good after surgical removal of a solitary tumour. They record such a case in a boy aged 10 years who remained well after operation, but the period of observation was only two months. In the present case the total duration of illness was six weeks.

Apart from the presence of a tumour there is generally little in the clinical picture to suggest an intestinal lesion, since lymphosarcoma, in marked contrast to carcinoma, rarely obstructs the bowel but, as in the present case, generally expands it. 
Ullman and Abeshouse attribute dilatation to destruction of muscle fibres combined with early loss of the nerve plexus in the submucosa. In our case it is remarkable, in view of the extensive intestinal tumour growth, that there were no clinical symptoms of alimentary disease apart from the single, brief attack of abdominal pain soon after the onset of illness. It is known, however, that in adults with excessive lymphoid hyperplasia of the intestinal tract, clinical symptoms may be very meagre, provided there is no intestinal obstruction or ulceration (Wells and Maver, 1904). SimpsonSmith, on clinical analysis of his material, found two facts of diagnostic importance: the high incidence of palpable abdominal tumour, and general deterioration with loss of weight, pallor, and exhaustion, all features of the case under discussion.

In view of the indefinite history and the rarity of intestinal neoplasms in childhood, it is not surprising that few diagnoses are made on clinical findings alone. Only two of ninety-one cases collected by Ullman and Abeshouse were correctly diagnosed without $x$-ray examination or biopsy. Occasionally lymphosarcoma of the bowel, especially the polypoid variety, presents as an intussusception. Thus in the series reported by Cutler et al., lymphosarcoma had induced intussusception in three cases, including the youngest, an infant aged 4 months. In Kasemeyer's (1912) series of 284 intussusceptions due to tumour, twenty-six had lymphosarcoma as the starting point. It is extremely rare to find multiple lymphoid tumours of the small bowel in childhood, though a somewhat similar condition with multiple foci of lymphoid hyperplasia is described in adults under the name of pseudo-leukaemia gastrointestinalis. Here the mucosa of stomach and bowel is greatly thickened and thrown into large folds resembling cerebral convolutions. Such cases are recorded by Wells and Maver, and Biggs and Elliott (1924). In true lymphatic leukaemia, also, widespread infiltration of the gastro-intestinal tract may occur, as described by Boikan (1931) and Pearson et al. (1943). These cases generally show an associated lymphoid infiltration of lymph glands and viscera.

The kidneys in the present case presented a remarkable appearance and resembled symmetrical bilateral renal tumours. Ewing (1922) states that the kidney is a favourite seat of metastasis in lymphosarcoma, which may take the form of minute foci, diffuse infiltrations, or bulky masses. Most of the scanty recorded cases of massive renal involvement are in men or youths. Thus, Symmers (1918) saw examples of massive symmetrical enlargement of the kidneys in three cases of lymphosarcoma, all in men, in two of whom the thymus appeared to be the site of origin, while in the third the small bowel had multiple annular tumours. Cohnheim (1865) describes a similar renal lesion in his original adult case of pseudo-leukaemia. Bilateral renal enlargement closely similar to that under discussion is recorded by Turner (1878) in a boy of 15 with a lymphosarcoma of the thymus and general glandular and splenic enlargement. He aptly compares the creamy-white colour of the tumour growth to that of a parsnip. Gouget and Thibaut (1911) saw massive renal infiltration in lymphatic leukaemia in a boy aged 16 with enlarged cervical glands and a growth in the mediastinum. Both organs were of uniform whitish colour with petechial haemorrhages and resembled bilateral tumours as in the present case. All these authors remark on the fact that the urine was normal, except for a trace of albumin and sometimes the presence of a few granular or hyaline casts and epithelial cells.

The blood picture in our case showed no evidence of lymphatic leukaemia, even in the terminal stage, but the close relation between lymphosarcoma and lymphatic leukaemia is well recognized. Gall and Mallory (1942), in a large series of cases, frequently saw the development of lymphatic leukaemia in patients with lymphosarcoma, and this was found by Cutler et al. in two of their five juvenile cases with intestinal tumours. The similarity of the cellular infiltration in the bowel, glands, and various organs in lymphosarcoma and lymphatic leukaemia, and the close resemblance of the lymphosarcoma cells in the tissues to the lymphocytes in the blood, serve to confirm the near relationship of the two diseases. Willis sums up the position in the dictum, 'Except for the demonstrable presence or absence of malignant cells in the circulating blood, there is no difference between lymphosarcoma and lymphatic leukaemia in either gross or microscopic pathology.'

\section{Summary}

A case of intestinal lymphosarcoma in a boy is described which proved fatal in six weeks from the clinical onset. At autopsy, widespread tumour growth was found in the intestines, kidneys, mesenteric glands, and bones. Clinical symptoms of gastro-intestinal or renal disease had been negligible.

The diagnosis of intestinal lymphosarcoma should be considered in a child with an abdominal tumour accompanied by rapidly progressive loss of weight, pallor, and lassitude.

Thanks are due to Professor G. B. Fleming for permission to consult the case record, to Mrs. Hamilton (C. Brown Kelly) for the drawings, and to Dr. A. H. T. Robb-Smith to whom sections were referred.

\section{REFERENCES}

Barrett, A. M. (1944). J. Path. Bact., 56, 133.

Biggs, A. D., and Elliott, A. R. (1924). J. Amer. med. Ass., 83, 178

Boikan, W. S. (1931). Arch. intern. Med., 47, 42.

Boyd, W. (1947). Surgical Pathology. Saunders. Philadelphia and London. Sixth Edit., p. 592.

Cohnheim, J. (1865). Virchow's Arch., 33, 451.

Cutler, G. D., Stark, R. B., and Scott, H. W., Jr. (1945). New Engl. J. Med., 232, 665. 
Ewing, J. (1913). J. Med. Research, 28, 1. (1922). Neoplastic Diseases. Saunders. Philadelphia and London. Second Edit., pp. 387-90.

Gall, E. A., and Mallory, T. B. (1942). Amer. J. Path., 18, 381.

Gouget, -., and Thibaut, - (1911). Bull. Mém. Soc. Méd. Hôp. Paris, 31, 583.

Jopson, J. H., and White, C. Y. (1901). Amer. J. med. Sci., $122,807$.

Kasemeyer, E. (1912). Dtsch. Z. Chir., 118, 205.

Lendrum, A. C. (1947). In Recent Advances in Clinical Pathology, edited by S. C. Dyke. Churchill, London. p. 452.

Libman, E. (1900). Amer. J. med. Sci., 120, 309.

Munson, C. L., and Marx, H. E. (1946). Delaware State med. J., 18, 153.
Pearson, B., Stasney, J., and Pizzolato, P. (1943). Arch. Path., 35, 21.

Robb-Smith, A. H. T. (1938). J. Path. Bact., 47, 457.

Simpson-Smith, A. (1938). Brit. J. Surg., 26, 429.

Stern, C. (1901). Berlin Klin. Wschr., 14, 1043.

Symmers, D. (1918). Arch. intern. Med., 21, 237.

Turner, F. C. (1878). Trans. Path. Soc., London, 29, 342.

Ullman, A., and Abeshouse, B. S. (1932). Ann. Surg., 95, 878.

Warthin, A. S. (1915). Arch. intern. Med., 15, 444.

Wells, H. G., and Maver, M. B. (1904). Amer. J. med. Sci., 128, 837.

Willis, R. A. (1948). Pathology of Tumours. Butterworth, London. pp. $765,768,778$. 